\title{
Pseudohypoaldosteronism type 2
}

INSERM

\section{Source}

INSERM. (1999). Orphanet: an online rare disease and orphan drug data base.

Pseudohypoaldosteronism type 2. ORPHA:757

A rare genetic form of hypertension characterized by hyperkalemia, mild hyperchloremic metabolic acidosis, normal or elevated aldosterone, low renin, with normal renal glomerular filtration rate (GFR). 